\title{
CORRESPONDENCE
}

\section{Does a Microphthalmia-Associated Transcription Factor-Pigment Epithelium-Derived Factor Axis Exist in All Types of Pigment Cells?}

\author{
Xiaoyin Ma and Ling Hou
}

From the Laboratory of Developmental Cell Biology and Disease, School of Ophthalmology and Optometry and Eye Hospital, and the State Key Laboratory Cultivation Base and Key Laboratory of Vision Science of Ministry of Health and Zhejiang Provincial Key Laboratory of Ophthalmology, Wenzhou Medical University, Wenzhou, China

\section{To the Editor-in-Chief:}

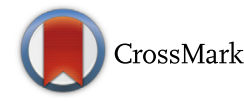

In a recent article ${ }^{1}$ published and highlighted ${ }^{2}$ in the correspondence section in The American Journal of Pathology, Dadras et al $^{1}$ found that, in primary melanocytes and melanoma cells, the microphthalmia-associated transcription factor (MITF) regulates the expression of the geneencoding pigment epithelium-derived factor (PEDF). In addition, the authors suggested that PEDF plays critical roles in melanoma progression. Consistent with these results, Fernández-Barral et $\mathrm{al}^{3}$ earlier reported that MITF regulates the expression of PEDF in melanoma cells and inhibits melanoma metastasis. On the basis of the similar results published by two independent research groups, there seems to be little doubt that the MITF-PEDF axis regulates melanoma metastasis.

PEDF was originally discovered not in melanoma cells but in a different melanin-bearing, non-transformed cell type, human retinal pigment epithelial cells. ${ }^{4}$ We have previously shown that MITF regulates PEDF in retinal pigment epithelial cells to inhibit their migration. ${ }^{5}$ Because retinal pigment epithelial cells are embryologically derived from the optic neuroepithelium, and melanoma cells, ultimately from the neural crest, it follows that pigment cells of distinct embryological origin and functions are apparently both under the control of the same transcription factor or target gene. Nevertheless, several important questions still need to be addressed.

First, are PEDF functions limited to migration of melanoma cells or can they be extended to melanoblasts and melanocytes during development or in wound healing and vitiligo repigmentation? It is well known that MITF mutations in humans are associated with Waardenburg syndrome type 2, a syndrome characterized by skin hypopigmentation and ocular pigmentation defects. ${ }^{6}$ Indeed, MITF plays important roles in melanoblast migration, and we recently found that it regulates melanoblast migration by repressing the melanoma cell adhesion molecule. ${ }^{7}$ Involvement of PEDF in this pathway, however, has not yet been demonstrated.

Second, how does PEDF inhibit melanoma metastasis? Both Dadras et $\mathrm{al}^{1}$ and Fernández-Barral et $\mathrm{al}^{3}$ clearly show a role for MITF to regulate PEDF, but how PEDF inhibits migration remains open. Because our earlier work ${ }^{5}$ identified impairment of microtubule dynamics as at least one of the mechanisms underlying migration inhibition, it is tempting to speculate that a similar mechanism might be operating in melanoma.

In summary, we do believe that the MITF-PEDF axis plays important roles in pigment cell migration. The above considerations make it important now to identify the underlying mechanisms by which PEDF controls melanoma metastasis.

\section{Acknowledgment}

We thank Dr. Heinz Arnheiter for thoughtful comments on the manuscript.

\section{References}

1. Dadras SS, Lin RJ, Razavi G, Kawakami A, Du J, Feige E, Milner DA, Loda MF, Granter SR, Detmar M, Widlund HR, Horstmann MA,

\footnotetext{
Supported by the Zhejiang Provincial Natural Science Foundation grants LZ12C12001 and LQ13H120004.

Disclosures: None declared.

Address correspondence to Ling Hou, Ph.D., Laboratory of Developmental Cell Biology and Disease, Wenzhou Medical University, 270 Xueyuanxilu, Wenzhou, China. E-mail: 1hou88@aliyun.com.
} 
Fisher DE: A novel role for microphthalmia-associated transcription factor regulated pigment epithelium-derived factor during melanoma progression. Am J Pathol 2015, 185:252-265

2. Jiménez Cuenca B, Fernández-Barral A, Luis Orgaz J: Microphthalmiaassociated transcription factor: a central regulator of pigment epitheliumderived factor controlling human melanoma progression. Am J Pathol 2015, 185:2069-2070

3. Fernández-Barral A, Orgaz JL, Baquero P, Ali Z, Moreno A, Tiana M, Gómez V, Riveiro-Falkenbach E, Cañadas C, Zazo S, Bertolotto C, Davidson I, Rodríguez-Peralto JL, Palmero I, Rojo F, Jensen LD, del Peso L, Jiménez B: Regulatory and functional connection of microphthalmia-associated transcription factor and anti-metastatic pigment epithelium derived factor in melanoma. Neoplasia 2014, 16:529-542
4. Tombran-Tink J, Johnson LV: Neuronal differentiation of retinoblastoma cells induced by medium conditioned by human RPE cells. Invest Ophthalmol Vis Sci 1989, 30:1700-1707

5. Ma X, Pan L, Jin X, Dai X, Li H, Wen B, Chen Y, Ma A, Qu J, Hou L: Microphthalmia-associated transcription factor acts through PEDF to regulate RPE cell migration. Exp Cell Res 2012, 318: 251-261

6. Arnheiter H: The discovery of the microphthalmia locus and its gene, Mitf. Pigment Cell Melanoma Res 2010, 23:729-735

7. Rao C, Su Z, Li H, Ma X, Zheng X, Liu Y, Lu F, Qu J, Hou L: Microphthalmia-associated transcription factor regulates skin melanoblast migration by repressing the melanoma cell adhesion molecule. Exp Dermatol 2015, [Epub ahead of print] doi:10.1111/exd.12835 